\title{
Ischaemic stroke and liver fibrosis
}

Author Affiliation: UCL Institute of Cardiovascular Science, UCL, London

Running title: Ischaemic stroke and liver fibrosis

Word count: 1505

Corresponding Address:

Dr Ann Walker PhD

UCL Institute of Cardiovascular Science

UCL

Rayne Building, 5 University Street

London WC1E 6JF, UK

Tel: +44 (0)20 76796279

Email: ann.walker@ucl.ac.uk

Key words: ischaemic stroke; liver stiffness; liver fibrosis; liver steatosis; non-alcoholic fatty liver disease, NAFLD; non-alcoholic steatohepatitis; NASH; transient elastography; PNPLA3; ABO; single nucleotide polymorphism; SNP; pathogenesis 


\section{Editorial}

\section{Ischaemic stroke and liver fibrosis}

Stroke is the sudden onset of acute neurological injury arising as a result of cerebral haemorrhage or ischaemia. It is common and serious, with 16.9 million incident strokes deduced to have occurred in 2010 causing 5.9 million deaths worldwide. It is also a major cause of disability, incurring a significant healthcare burden. Ischaemic stroke is responsible for about $80 \%$ of cases in the developed world. Focal ischaemia results from blockage of a blood vessel by a thrombus or embolus, preventing blood supply to a region of the brain.(1) Brain tissue is exquisitely sensitive to interruption of blood flow which delivers glucose and oxygen.

Stroke and other cardiovascular diseases have been associated with various biomarkers and parameters of liver disease. Non-alcoholic liver disease (NAFLD) is the most common cause of liver dysfunction, affecting $\sim 20-30 \%$ of adults in Western countries. It spans pathologies ranging from simple fatty liver (steatosis; hepatocellular triglyceride content $>5 \%$ ) to necro-inflammatory nonalcoholic steatohepatitis (NASH), including scarring (fibrosis) and the formation of nodules surrounded by fibrotic bands (cirrhosis). For a diagnosis of NAFLD, secondary causes and excess alcohol consumption ( $\geq 30 \mathrm{~g} /$ day for men and $\geq 20 \mathrm{~g}$ for women) must be excluded.(2) NAFLD is associated with the conditions that define metabolic syndrome (abdominal obesity, high blood pressure, high fasting glucose, high triglycerides and low HDL levels) and hence patients, particularly those with NASH, may be at increased risk of cardiovascular disease, due to shared risk factors.(3) NAFLD and especially NASH may exacerbate hepatic and systemic insulin resistance, leading to dyslipidaemia, atherogenesis and increased cardiovascular disease risk. Remarkably, in NAFLD, cardiovascular disease is more commonly responsible for death than is liver disease. $(2 ; 4 ; 5)$ The relationship between ischaemic stroke and liver stiffness, as an indicator of hepatic fibrosis, is examined in the paper published by Kim and colleagues in this issue of Atherosclerosis.(6) These authors investigated 295 patients admitted with acute ischaemic stroke compared to 1,942 control subjects having a medical check-up, in Seoul, Korea. The most common causes of stroke were cardioembolism (39.5\%), more than two causes (26.2\%) and large artery atherothrombosis (12.9\%). Patients with chronic viral hepatitis or alcohol consumption of $>40 \mathrm{~g} /$ day for $>5$ years were excluded. Liver stiffness was assessed non-invasively by ultrasound investigation using transient elastography; values $<5.6 \mathrm{kPa}$ were taken as within the normal range. Liver steatosis was also assessed during the same examination via attenuation of the ultrasound signal as the controlled attenuation parameter (CAP); values $>250 \mathrm{~dB} / \mathrm{min}$ were taken as an indicator of fatty liver.

While normal liver stiffness was observed in a greater proportion of the control group than the stroke group ( $90.6 \%$ vs. $69.2 \%, \mathrm{p}<0.001)$ and a greater proportion of the stroke group had significant liver fibrosis ( $>8 \mathrm{kPa}$ ) than the control group ( $9.2 \% \mathrm{vs.} 1.8 \%, \mathrm{p}<0.001)$, the proportion with fatty liver (defined by CAP) did not differ between the groups ( $42.4 \%$ vs. $41.0 \%, p=0.665$ ). Similarly, the mean liver stiffness of the stroke group was greater than that of the control group (5.6 vs. $4.1 \mathrm{kPa}$, $p<0.001)$, whereas the mean CAP value did not differ between the groups $(242.1 \mathrm{vs} .243 .5 \mathrm{~dB} / \mathrm{m}$, $\mathrm{p}=0.646$ ). After stratifying according to either being overweight ( $\mathrm{BMI} \leq \mathrm{or}>25)$, having fatty liver (CAP $\leq$ or $>250$ ) and the presence or absence of metabolic syndrome, mean liver stiffness remained significantly greater in all stroke vs. control subgroups. 
The severity of fibrosis was compared between stroke and control groups using multiple logistic regression analysis. The odds ratio (OR) for ischaemic stroke was 1.268 (95\% confidence intervals $1.183-1.358, p<0.001)$ per $1 \mathrm{kPa}$ increase in liver stiffness. OR for ischaemic stroke in the context of mild fibrosis was $4.308(2.680-6.925, p<0.001)$ and of significant fibrosis was 12.033 (5.180-27.948, $\mathrm{p}<0.001)$, versus no fibrosis. Consistent results were obtained after propensity-score matched analysis of the subset of 189 cases and 189 controls: the OR for ischaemic stroke was 1.804 (1.461$2.230, p<0.001)$ per $1 \mathrm{kPa}$ increase; OR for ischaemic stroke in the context of mild fibrosis (5.6-8.0 $\mathrm{kPa}$ ) was $4.577(2.354-8.900, \mathrm{p}<0.001)$ and of significant fibrosis was $13.184(3.127-55.645, \mathrm{p}<0.001)$, versus no fibrosis.

The study population was also stratified using serum alanine aminotransferase (ALT) levels, as a marker of hepatocellular damage, into patients with normal ALT levels and those with levels above the upper normal limit of $0.67 \mu \mathrm{kat} / \mathrm{L}(>40 \mathrm{IU} / \mathrm{L})$. In patients with normal ALT levels, OR for ischaemic stroke was $1.708(1.472-1.982, p<0.001)$ per $1 \mathrm{kPa}$ increase in liver stiffness. OR for ischaemic stroke in the context of mild fibrosis was $4.661(2.609-8.325, \mathrm{p}<0.001)$ and of significant fibrosis was 6.805 (2.321-19.950, $p<0.001$ ), versus no fibrosis. In patients with ALT levels above the normal range, risk of ischaemic stroke was again associated with liver stiffness when analysed as a categorical variable, but not when analysed as a continuous variable. This may reflect previous observations that in patients with chronic hepatitis B, elevated ALT may be associated with over-estimation of liver stiffness by TE.(7)

Overall, this study $(6)$ is the first to show an association between the degree of liver fibrosis and risk of ischaemic stroke. This chimes with the previous observation that only NAFLD patients with fibrosis stage 3-4 (irrespective of the NAFLD activity score) had increased cardiovascular mortality, compared to a reference population.(5) Three previous studies (total subjects 2,241) have assessed stroke or transient ischaemic attack (excluding cerebral haemorrhage) in a prospective fashion, in subjects diagnosed with NAFLD by ultrasonography. Meta-analysis found stroke risk was higher in the group with NAFLD vs. the group without NAFLD (relative risk 2.09; 95\% Cl 1.46-2.98, p<0.001).(8)

Genetic risk factors have been proposed to predispose to NAFLD via four pathways, influencing glucose metabolism and insulin sensitivity, steatosis, steatohepatitis and the development of fibrosis.(9) Kim and colleagues did not investigate genetic risk factors for either stroke or NAFLD (and large studies would be required for this); however, there is an apparent lack of overlap between genome-wide significant associations for ischaemic stroke and NAFLD.(6;9-12) Several putative modifier variants have been reported for liver steatosis or histological evidence of NAFLD and a few genome-wide significant associations have been replicated. The s738409 variant in PNPLA3 predicting isoleucine to methionine substitution (lle148Met) has been robustly associated with serum biochemical markers of NAFLD and hepatic steatosis. The lle148Met protein has impaired triglyceride hydrolysis activity, coating hepatic lipid droplets and blocking access of other lipolytic enzymes, leading to accumulation of hepatic triglyceride.(13) Carriers of Ile148Met also secreted less of the large, triglyceride - rich very low density lipoprotein $\left(\mathrm{VLDL}_{1}\right)$ particles from the liver than did wild type lle148 homozygotes, for any given liver fat content.(14). For ischaemic stroke, genome-wide significant associations with specific stroke subtypes suggest clues to mechanism: PITX2 and ZFHX3 were associated with cardioembolic stroke, consistent with their original association with atrial fibrillation.(11) The HDAC9 and 9p21 associations were specific to large vessel stroke. $A B O$ (encoding $A B O$ blood group) was associated with all ischaemic stroke and large vessel and cardioembolic stroke.(10) The $A B O$ single nucleotide polymorphism (SNP), rs505922, was previously associated with levels of the clotting factors, von Willebrand factor and Factor VIII, consistent with a thrombotic mechanism. However, SNPs in the $A B O$ locus have widespread 
pleiotropic effects upon a range of cardiometabolic traits. For example, the lead ischaemic stroke SNP, rs532436, has been associated with red blood cell traits, lower haematocrit and haemoglobin, so in the case of a partly blocked artery, lower oxygen transport capacity of blood could be a potential contributing mechanism. $(10 ; 15 ; 16)$ In line with this, a study of $\sim 50,000$ subjects with stroke and 150,000 controls associated ischaemic stroke with iron deficiency anaemia.(17)

While liver biopsy is the gold standard for histological evaluation of NAFLD, allowing assessment of liver architecture, it is invasive and has potential for sampling error. Transient elastography is a widely used, non-invasive reference technique; 10 repeated measurements were made and measurements with interquartile range $>30 \%$ of median were rejected.(6) However, it may be unreliable for patients with high $\mathrm{BMI}$ and/or high thoracic fold thickness and the presence of steatosis may cause false positive results. $(2 ; 18)$ The CAP value can detect steatosis, is non-invasive and can be performed alongside transient elastography; however its discrimination of histological grades of steatosis is limited and more data are required to define its use.(19)

The findings of Kim and colleagues (6) merit further validation and replication in large, highly phenotyped prospective observational cohorts with hepatic, cardiometabolic and stroke - related data, genotype and other "-omics" measurements. The potential overlap between genetic risk factors, signalling pathways affected by genetic variants and their tissue expression patterns, along with causality, remain fertile ground for exploration. These observations on Korean patients should be investigated across cohorts of different ethnicities. If confirmed, they suggest a number of interesting questions and avenues. Firstly, is the association of liver stiffness with ischaemic stroke due only to shared risk factors? Secondly, the role of simple steatosis vs. NASH remains to be resolved in stroke risk: does hepatic inflammation add to stroke risk? Thirdly, would improvement in fatty liver disease reduce the risk of ischaemic stroke? Exploration of the relationship between ischaemic stroke risk and liver fibrosis has potential for utility, as well as providing insight into the underlying biological mechanisms. 
Reference List

(1) Hankey GJ. Stroke. Lancet 2017 Feb 11;389(10069):641-54.

(2) EASL-EASD-EASO Clinical Practice Guidelines for the management of non-alcoholic fatty liver disease. J Hepatol 2016 Jun;64(6):1388-402.

(3) Lawlor DA, Callaway M, Macdonald-Wallis C, Anderson E, Fraser A, Howe LD, et al. Nonalcoholic fatty liver disease, liver fibrosis, and cardiometabolic risk factors in adolescence: a cross-sectional study of 1874 general population adolescents. J Clin Endocrinol Metab 2014 Mar;99(3):E410-E417.

(4) Targher G, Day CP, Bonora E. Risk of cardiovascular disease in patients with nonalcoholic fatty liver disease. N Engl J Med 2010 Sep 30;363(14):1341-50.

(5) Ekstedt M, Hagstrom H, Nasr P, Fredrikson M, Stal P, Kechagias S, et al. Fibrosis stage is the strongest predictor for disease-specific mortality in NAFLD after up to 33 years of follow-up. Hepatology 2015 May;61(5):1547-54.

(6) Kim SU et al. Liver fibrosis with transient elastography is an independant risk factor for ischaemic stroke. Atherosclerosis, in press 2017

(7) Kim SU, Han KH, Ahn SH. Transient elastography in chronic hepatitis B: an Asian perspective. World J Gastroenterol 2010 Nov 7;16(41):5173-80.

(8) Mahfood HT, Hamdeh S, Kanmanthareddy A, Alla VM. Nonalcoholic fatty liver disease and the risk of clinical cardiovascular events: A systematic review and meta-analysis. Diabetes Metab Syndr 2016 Dec 15.

(9) Anstee QM, Day CP. The genetics of NAFLD. Nat Rev Gastroenterol Hepatol 2013 Nov;10(11):645-55.

(10) Malik R, Traylor M, Pulit SL, Bevan S, Hopewell JC, Holliday EG, et al. Low-frequency and common genetic variation in ischemic stroke: The METASTROKE collaboration. Neurology 2016 Mar 29;86(13):1217-26.

(11) Traylor M, Farrall M, Holliday EG, Sudlow C, Hopewell JC, Cheng YC, et al. Genetic risk factors for ischaemic stroke and its subtypes (the METASTROKE collaboration): a meta-analysis of genome-wide association studies. Lancet Neurol 2012 Nov;11(11):951-62.

(12) Severson TJ, Besur S, Bonkovsky HL. Genetic factors that affect nonalcoholic fatty liver disease: A systematic clinical review. World J Gastroenterol 2016 Aug 7;22(29):6742-56.

(13) Smagris E, BasuRay S, Li J, Huang Y, Lai KM, Gromada J, et al. Pnpla3I148M knockin mice accumulate PNPLA3 on lipid droplets and develop hepatic steatosis. Hepatology 2015 Jan;61(1):108-18.

(14) Pirazzi C, Adiels M, Burza MA, Mancina RM, Levin M, Stahlman M, et al. Patatin-like phospholipase domain-containing 3 (PNPLA3) I148M (rs738409) affects hepatic VLDL secretion in humans and in vitro. J Hepatol 2012 Dec;57(6):1276-82. 
(15) McLachlan S, Giambartolomei C, White J, Charoen P, Wong A, Finan C, et al. Replication and Characterization of Association between ABO SNPs and Red Blood Cell Traits by MetaAnalysis in Europeans. PLoS One 2016;11(6):e0156914.

(16) Williams FM, Carter AM, Hysi PG, Surdulescu G, Hodgkiss D, Soranzo N, et al. Ischemic stroke is associated with the ABO locus: the EuroCLOT study. Ann Neurol 2013 Jan;73(1):16-31.

(17) Chang YL, Hung SH, Ling W, Lin HC, Li HC, Chung SD. Association between ischemic stroke and iron-deficiency anemia: a population-based study. PLoS One 2013;8(12):e82952.

(18) Petta S, Maida M, Macaluso FS, Di M, V, Camma C, Cabibi D, et al. The severity of steatosis influences liver stiffness measurement in patients with nonalcoholic fatty liver disease. Hepatology 2015 Oct;62(4):1101-10.

(19) de Ledinghen V, Vergniol J, Capdepont M, Chermak F, Hiriart JB, Cassinotto C, et al. Controlled attenuation parameter (CAP) for the diagnosis of steatosis: a prospective study of 5323 examinations. J Hepatol 2014 May;60(5):1026-31. 